\title{
Eficacia pronóstica de la determinación de los receptores moleculares ERa y Her2-neu en tumores mamarios caninos
}

\author{
Benítez, J.S.; Alfaro, N.*; Insfrán, R.M.; Merlo, W.A.; Rosciani, A.S.
}

Servicio de Diagnóstico Histopatológico y Citológico, Facultad de Ciencias Veterinarias, Universidad Nacional del Nordeste, Sargento Cabral 2139, Corrientes (3400), Argentina. Tel. (03783) 425753. E-mail: arosciani@vet.unne. edu.ar. *Laboratorio de Biología Celular y Molecular, Facultad Veterinaria, Universidad Nacional del Litoral.

\begin{abstract}
Resumen
Benítez, J.S.; Alfaro, N.; Insfrán, R.M.; Merlo, W.A.; Rosciani, A.S.: Eficacia pronóstica de la determinación de los receptores moleculares ER a y Her2-neu en tumores mamarios caninos. Rev. vet. 22: 2, 119-122, 2011. El objetivo del presente trabajo fue resaltar el valor que representa la determinación de receptores de estrógeno alfa (Er $\alpha)$ y del receptor tipo 2 del factor de crecimiento epitelial (Her2-neu) en los tumores mamarios, así como el estadio clínico (EC) de las pacientes caninas al momento de la consulta, a fin de utilizar dichos datos para emitir un pronóstico. Se trabajó con muestras histopatológicas de 24 tumores mamarios de pacientes que concurrieron en forma espontánea al Hospital de Clínicas de la Facultad de Ciencias Veterinarias de la UNNE (Corrientes, Argentina) durante el período 2010-2011. Las pacientes se clasificaron de acuerdo a su EC en cinco grupos. La inmunomarcación de ER $\alpha$ arrojó los siguientes valores promedio: $58 \%$ para EC I, 52\% para EC II, $22 \%$ para EC III, $13 \%$ para EC IV y $12 \%$ para EC V. Por su parte, la marcación de Her2-neu mostró los siguientes valores promedio: $13 \%$ para EC I, $14 \%$ para EC II, $42 \%$ para EC III, $48 \%$ para EC IV y $50 \%$ para EC V. Se encontraron diferencias estadísticamente significativas entre los diferentes estadios clínicos, para ambos marcadores moleculares (ER $\alpha p=0,0026$; Her2-neu $p=0,0489$ ). Los resultados muestran una sensible disminución de la expresión de ER $\alpha$ a medida que aumenta la estadificación clínica, es decir, a medida que el tumor y el cuadro clínico de la paciente muestran mayores indicios de malignidad. Por el contrario, la expresión del receptor Her2-neu, se comporta a la inversa, aumentando conjuntamente con la estadificación clínica. Estos resultados evidencian la necesidad de realizar un análisis clínico exhaustivo de las pacientes con neoplasia, así como la inmunomarcación de ER $\alpha$ y Her2-neu de las muestras en forma rutinaria, ya que estas determinaciones permitirían emitir un pronóstico más certero y sugerir posibles terapias adecuadas a cada caso.
\end{abstract}

Palabras clave: perro, neoplasia mamaria, receptores ER $\alpha /$ Her2-neu, valor pronóstico.

\begin{abstract}
Benítez, J.S.; Alfaro, N.; Insfrán, R.M.; Merlo, W.A.; Rosciani, A.S.: Predictive effectiveness of the determination of two molecular receptors ER $\alpha$ and Her2-neu in canine mammary tumors. Rev. vet. 22: 2, 119-122, 2011. The aim of this paper is to highlight the value that represents the assessment of alpha estrogen receptors (ER $\alpha)$ and type 2 receptor of epithelial growing factor (Her2-neu) in mammary tumors and the consideration of canine patients' clinical stage (CS) at first consultation, in order to use these data to carry out a clinical prognostic. We worked with histopathological samples of breast tumors from 24 patients that spontaneously came to the Clinical Hospital of the School of Veterinary Medicine, UNNE (Corrientes, Argentina) during the period 2010-2011. Patients were classified according to their CS into five groups. ER $\alpha$ immunostaining showed the following average values: $58 \%$ for CS I, $52 \%$ for CS II, $22 \%$ for CS III, $13 \%$ for CS IV and $12 \%$ for CS V. At the same time, Her2-neu analysis showed the following average values: $13 \%$ for CS I, $14 \%$ for CS II, $42 \%$ for CS III, $48 \%$ for CS IV and $50 \%$ for CS V. Statistically significant differences were found for different clinical staging for both molecular markers (ER $\alpha p=0.0026$, Her2-neu $p=0.0489$ ). The results show a clear decrease in ER $\alpha$ expression with a simultaneous raise in clinical staging, meaning this that the tumor and the patient show incremented malignancy. On the other hand, the expression of Her2-neu receptor behaves conversely, increasing in conjunction with clinical staging. These results highlight the need of a thorough patient's
\end{abstract}


clinical analysis as well as a routine determination of ER $\alpha$ and Her2-neu since these findings allow to perform a confident prediction outcome and may help in the selection of appropriate therapies considering each individual case.

Key words: bitch, mammary neoplasia, receptors ER $\alpha$ / Her2-neu, prognostic value.

\section{INTRODUCCIÓN}

Los tumores mamarios caninos constituyen el tipo de neoplasia de presentación más frecuente en la especie canina. Afecta principalmente a las hembras enteras entre los 6 y 9 años de edad ${ }^{11}$, constituyendo las manifestaciones de tipo malignas cerca del $90 \%$ de los casos que concurren a la consulta en esta región ${ }^{14}$. El tiempo de supervivencia estimado de los pacientes tratados por neoplasias malignas oscila entre los 4 y 18 meses ${ }^{10}$. Por tal motivo, resulta de gran interés determinar factores evaluables que permitan emitir un pronóstico, orientar un tratamiento y generar nuevas posibles terapias.

La actividad de la glándula mamaria normal es regulada por hormonas esteroideas, como el estradiol, que ejercen su acción durante el ciclo estral estimulando el crecimiento y el desarrollo túbuloalveolar y ductal y la hiperplasia de los elementos secretorios ${ }^{15,17}$. Las neoplasias mamarias se consideran hormono dependientes ${ }^{4}$, por lo que se ha puesto mucha atención a las hormonas esteroides durante la génesis tumoral ${ }^{5,18}$. De hecho, el estradiol, juega un rol importante en la patogénesis de la neoplasia mamaria, ya que mediado por la unión a su receptor, promueve fenómenos de iniciación y promoción carcinogénica, a través de sus efectos mitogénicos sobre las células de la glándula ${ }^{16}$. Los receptores estrogénicos han sido descriptos en tumores mamarios malignos, así como en tumores mamarios benignos y en el tejido glandular normal ${ }^{2}$.

En seres humanos, los tumores ricos en receptores de estradiol responden a la terapia tradicional de ablación endócrina ${ }^{13}$, pero aquellos tumores que carecen de tales receptores no lo hacen y son considerados de peor pronóstico ${ }^{3}$. En general, las neoplasias con pronóstico desfavorable sobreexpresan el oncogen Her2-neu vinculado al desarrollo tumoral ${ }^{8}$. El mismo codifica una glucoproteína que actúa como receptora de membrana celular y capta las señales de proliferación, específicamente, al factor de crecimiento epitelial ${ }^{9}$.

El receptor Her2-neu es un componente normal expresado en distintos tipos de células tumorales. En el cáncer de mama, una fracción de las pacientes sobreexpresan al Her2-neu como parte del proceso de malignización. La sobreexpresión de Her2-neu implica un pobre pronóstico, debido a que señala un alto nivel de proliferación celular, un peor grado histológico, una mayor agresividad biológica, una menor sobrevida total y un mayor riesgo de metástasis ${ }^{8,9}$. En medicina humana, el interés de su determinación radica principalmente en la posibilidad de aplicar inmunoterapia dirigida contra Her2-neu, mediante el anticuerpo monoclonal trastuzumab (Herceptin $\left.{ }^{\circledR}\right)$ y la utilización de quimioterapia ${ }^{1}$.
Sin embargo, en la neoplasia mamaria canina, el rol del Her2-neu aún está pobremente entendido. Algunos autores expresan la inexistencia de correlación entre la sobreexpresión de este receptor y la progresión tumoral u otro factor de tipo pronóstico ${ }^{7}$, mientras que otros investigadores lo correlacionan solamente con el tipo histopatológico y no con la presencia de invasión local o metástasis regionales ${ }^{20}$.

Por su parte, el estadio clínico (EC) es una de las herramientas que permite determinar el alcance de la enfermedad y observar su posible evolución durante el seguimiento, con el fin de definir la estrategia de tratamiento más adecuada. Para su determinación se aplica la "Clasificación TNM" (OMS) ${ }^{13}$, siendo necesario para tal fin una adecuada exploración clínica del paciente y la realización de radiografías de tórax y ecografías de abdomen. Una vez obtenidos los datos, se calcula el estadio clínico, de acuerdo al tamaño del tumor (T), el compromiso de los ganglios linfáticos (N) y la posible presencia de metástasis en diferentes órganos (M), existiendo 5 categorías diferentes de acuerdo a lo citado.

El objetivo de este trabajo fue resaltar el valor que representa la determinación de los marcadores moleculares ER $\alpha$ y Her2-neu y del estadio clínico (EC) de las pacientes caninas al momento de la consulta, a fin de utilizar dichos datos para emitir un pronóstico preciso.

\section{MATERIAL Y MÉTODOS}

Se trabajó con muestras histopatológicas de tumores mamarios de 24 perras que concurrieron en forma espontánea al Hospital de Clínicas de la Facultad de Ciencias Veterinarias de la UNNE (Corrientes, Argentina) durante el período 2010-2011. La toma de muestras, el procesamiento (por medio de la técnica histológica clásica) y el diagnóstico histopatológico se llevó a cabo en el Servicio de Diagnóstico Histopatológico y Citológico de la misma Facultad. La inmunomarcación se realizó en el Laboratorio de Biología Celular y Molecular de la Facultad de Ciencias Veterinarias de la Universidad del Litoral (Esperanza, Argentina).

ER $\alpha$ y Her 2-neu se identificaron por medio de la inmunomarcación de muestras histopatológicas de los tumores mamarios. Se utilizó el kit de biotina-streptavidina-peroxidasa Supersensitive Immunodetection System, LP000-UL, Biogenex, CA, USA. En cuanto a la técnica de inmunomarcación, los cortes obtenidos fueron colocados sobre portaobjetos pre-tratados con poly-L-lisina y, luego de la inactivación de la peroxidasa endógena, las distintas muestras se incubaron con el anticuerpo primario: 
Tabla 1. Porcentajes promedio de marcación de ER $\alpha$ y Her2-neu en relación al estadio clínico.

\begin{tabular}{cccc}
\hline $\begin{array}{c}\text { estadio } \\
\text { clínico }\end{array}$ & ER $\alpha$ & Her2-neu & $\begin{array}{c}\text { cantidad de } \\
\text { pacientes }\end{array}$ \\
\hline I & 58 & 13 & 3 \\
II & 52 & 14 & 3 \\
III & 22 & 42 & 14 \\
IV & 13 & 48 & 2 \\
V & 12 & 50 & 2 \\
\hline
\end{tabular}

ER $\alpha$ : receptores de estrógeno alfa; Her2-neu: receptor tipo 2 del factor de crecimiento epitelial.

- ERa: anticuerpo monoclonal de ratón anti-humano, Estrogen Receptor Clon 1D5 Dako N1575. Patrón de marcación nuclear.

- Her2-neu: anticuerpo policlonal de conejo, c-erbB-b Oncoprotein Dako Code No. A0485. Patrón de marcación citoplasmático y de membrana.

Luego se realizó la incubación con el anticuerpo secundario marcado con biotina y a continuación se incorporó la peroxidasa-estreptavidina. El revelado se logró con el agregado de diaminobencidina (DAB) y finalmente se coloreó con hematoxilina como tinción de contraste. Todas las diluciones de anticuerpo primario, secundario, estreptavidina-peroxidasa, DAB se hicieron en buffer fosfato salino (PBS) pH 7. Todas las series incluyeron controles positivos y controles negativos. Para la interpretación de la inmunomarcación se contaron tres campos de cien células y se realizó un promedio de los recuentos obtenidos de núcleos positivos para el caso de ER $\alpha$ y de membranas y citoplasmas en el caso de Her2-neu.

La determinación del estadio clínico (EC) se realizó en base a los datos obtenidos de la exploración clínica y de los estudios complementarios correspondientes. Las pacientes se clasificaron de acuerdo a su estadio en cinco grupos (I - V). Con los datos obtenidos, se procedió a efectuar el análisis estadístico mediante el Software InfoStat versión 2008.

\section{RESULTADOS}

Las neoplasias estudiadas fueron diagnosticadas de acuerdo con la clasificación histológica de los tumores mamarios del perro ${ }^{10}$ : carcinoma simple $(n=6)$, carcinoma complejo $(\mathrm{n}=11)$, carcinoma en tumor mixto $(n=5)$ y sarcoma $(n=2)$. Todas ellas constituyen variedades malignas. Del total de 24 casos analizados en el presente trabajo, la cantidad de pacientes correspondientes a cada estadio clínico se consigna en la Tabla 1, en la cual figuran también los valores promedio obtenidos de la inmunomarcación de ER $\alpha$ y de Her2-neu.

La marcación de ER $\alpha$ mostró valores promedio más elevados en los estadios clínicos inferiores, es decir, en aquellos considerados favorables para la evolución y también una mayor intensidad de marcación nu- clear tanto en células epiteliales carcinomatosas, como también en células mioepiteliales (Figura 1).

La positividad a la inmunomarcación de receptores Her2-neu (Figura 2) fue en aumento en los estadios clínicos de pronóstico más desfavorable. La intensidad de marcación observada, no guardó relación con los porcentajes de células positivas en cada caso.

Los valores promedio de inmunomarcación de cada receptor, mostraron diferencias significativas entre los diferentes estadíos clínicos (ER $\alpha p=0,0026$; Her2-neu $p=0,0489$ ).

\section{DISCUSIÓN}

La inmunomarcación de ER $\alpha$ tanto en las células de la glándula mamaria normal así como en las neoplasias de tipo benignas y malignas, se observa en la región del núcleo celular, pero la diferencia radica en el número de células positivas y en la intensidad de la reacción. Estos resultados han sido comunicados ampliamente en medicina humana y pueden ser atribuidos a la actividad de diferenciación celular. Estudios anteriores en perros ${ }^{6,12,16}$ han reportado la existencia de mayores porcentajes de marcación en los tumores benignos que

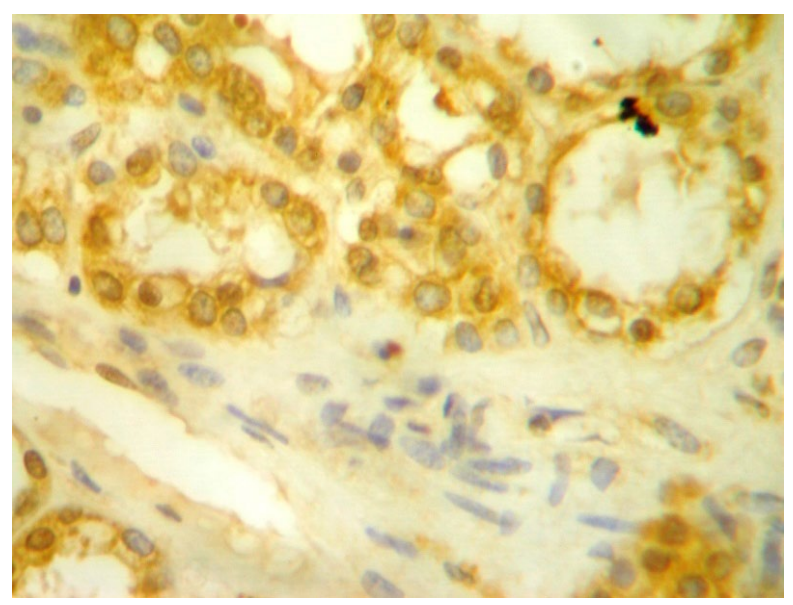

Figura 1. Inmunomarcación de ER $\alpha$. Se observan núcleos reactivos de células epiteliales carcinomatosas. 40x.

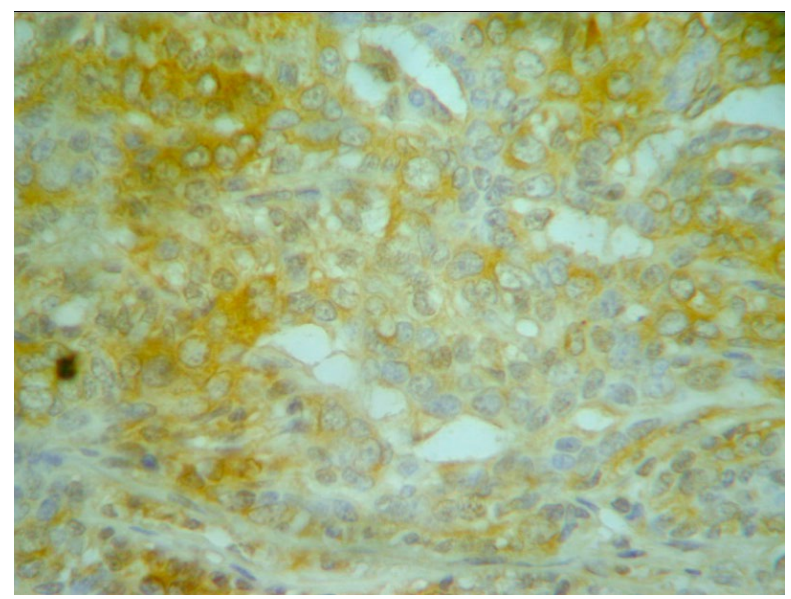

Figura 2. Inmunomarcación de Her2-neu. Se observa marcación citoplasmática y de membrana. 40x. 
en los malignos, lo que indicaría que estas neoplasias mantienen algún tipo de mecanismo regulatorio hormonal y está asociado a una relativamente lenta actividad de proliferación celular ${ }^{19}$.

Los resultados aquí obtenidos, exclusivamente de neoplasias malignas, muestran una sensible disminución en los porcentajes de expresión de ER $\alpha$ a medida que aumenta la estadificación clínica, es decir, a medida que el tumor y el cuadro clínico de la paciente muestran mayores indicios de malignidad.

En medicina veterinaria se observan resultados dispares con respecto al valor pronóstico de Her2-neu, pero nuestros hallazgos muestran un aumento de la marcación de tales receptores en forma concomitante con la estadificación clínica, demostrando en este caso, ser un indicador desfavorable para la evolución de las pacientes, concordando con lo que ocurre en los tumores mamarios de la mujer.

Por lo expuesto, se desprende la necesidad de realizar un análisis clínico exhaustivo de las pacientes caninas con neoplasia y determinar ambos marcadores en forma rutinaria, ya que estos parámetros permitirían realizar pronósticos más certeros y la posible aplicación de diferentes terapias más apropiadas a cada caso.

\section{REFERENCIAS}

1. Bruck P, Vilches Cisneros N, Ramos E, Barboza O, Ancer J, Flores JP. 2006. Expresión del Her2-neu en el adenocarcinoma ductal de la glándula mamaria: correlación con parámetros histopatológicos y expresión de receptores estrogénicos en pacientes mexicanas. Ginecol Obstet Mex 74: 516-522.

2. Corrada Y, Gobello C. 2001. Acromegalia del diestro en la perra. Analecta Vet 21: 57-62.

3. Donnay I, Devleeschower N, Wouters-Ballman P. 1996, Relationship between receptors for epidermal growth factor and steroid hormones in normal, dysplastic and neoplastic canine mammary tissues. Res Vet Sci 60: 251-254.

4. Fosum TW, Hedlund CS, Huilse DA, Johnson AL, Seim AL, Willard MD, Carrol GL. 1999. Cirugía en pequeños animales, Interamericana, Buenos Aires, $1632 \mathrm{p}$.

5. Hellmén E. 1996. The patogenesis of canine mammary tumors. Cancer J 9: 282-286.

6. Inaba T, Takahashi N, Matsuda H, Imori T. 1984. Estrogen and progesterone receptors and progesterone metabolism in canine mammary tumors. Jap J Vet Sci 46: 797-803.

7. Kim JH, Im KS, Kim NH, Yhee JY, Nho WG, Sur JH. 2011. Expression of Her-2 and nuclear localization of Her-3 protein in canine mammary tumors: histopathological and immunohistochemical study. Vet $J$ 189: 318-322.
8. Laboratorios Roche. 2005. Cáncer de mama. Pruebas HER-2/neu. On line: http://www.roche.com.ar/salud/ oncologia/c.mama/oncologia_mamall.asp.

9. Lamelas M, Vázquez J, González L, Rodil A, Vérez $\mathbf{P}$, Vizoso F, Raigoso P. 2002. Determinación de c.erbB-2/ neu en cáncer de mama: análisis comparativo de la determinación mediante análisis inmunoenzimático (ELISA) e inmunohistoquímico. On line: http://conganat.uninet.edu/ autores/trabajos/T024/.

10. Misdorp W, Else RW, Hellmén E, Lipscomb TP. 1999. Histological classification of mammary tumors of the dog and the cat, Publ. Armed Forces Institute of Pathology \& WHO, Washington (USA) 7: 58-59.

11. Moulton JE. 1990. Tumors of the mammary gland. In: $T u$ mors in domestic animals (Moulton JE, ed.), $3^{\text {rd }}$ ed., California Press, Berkeley, p. 518-553.

12. Nieto A, Pena L, Perez MD, Sanchez MA, Flores JM, Castano M. 2000. Immunohistologic detection of estrogen receptor alpha in canine mammary tumors: clinical and pathologic associations and prognostic significance. Vet Pathol 37: 239-247.

13. Owen L. 1980. TNM. Classification of tumors in domestic animals. Publ. World Health Organization, Geneva, p. 4647.

14. Rooney MT, Henry JB. 1993. Marcadores moleculares de las neoplasias malignas. Diagnóstico y tratamiento clinico, $9^{\mathrm{a}}$ ed., Masson, Barcelona, p. 293-315.

15. Rosciani AS, Merlo WA, Guaimás Moya LE. 2003. Aplicación de la clasificación histopatológica de la OMS (1999) a los tumores mamarios caninos. Anales XXIV Sesión Com Cientif Fac Vet UNNE (Corrientes, Argentina), p. 5.

16. Rutteman GR, Withrow SJ, MacEwen EG. 2001. Tumors of the mammary gland. In: Small Animal Clinical Oncology (Withrow SJ, MacEwen EG, ed), 3rd ed., Saunders, Philadelphia, p. 455-477.

17. Torres CG. 2005. Función del estradiol en el desarrollo de la neoplasia mamaria canina. Monogr Electrón Patol 2: 94-111.

18. Van Garderen E, Schalken JA. 2002. Morphogenic and tumorigenic potentials of the mammary growth hormone/ growth hormone receptor system. Mol Cell Endocrinol 197: 153-165.

19. Yang WY, Liu CH, Chang CJ, Lee CC, Chang KJ, Lin CT. 2006. Proliferative activity, apoptosis and expression of oestrogen receptor and Bcl-2 oncoprotein in canine mammary gland tumours. J Comp Path 134: 70-79.

20. Zaidan Dagli ML. 2008. The search for suitable prognostic markers for canine mammary tumors: a promising outlook. Vet $J$ 177: 3-5. 